\title{
Multimodality Treatment for Thymic Carcinoma: Review of 11 Cases at a Single Institute
}

\author{
Hisao Higo ${ }^{1}$, Keiichi Fujiwara ${ }^{1}$, Go Makimoto ${ }^{1}$, Yuhei Tokimasa ${ }^{1}$, Nobuhisa Kameyama ${ }^{1}$, \\ Mizuho Matsushita ${ }^{1}$, Kammei Rai ${ }^{1}$, Ken Sato ${ }^{1}$, Takuo Shibayama ${ }^{1}$, Toshiro Yonei ${ }^{1}$, Akio Andou ${ }^{2}$, \\ Toshio Sato ${ }^{1}$
}

${ }^{1}$ Department of Respiratory Medicine, National Hospital Organization Okayama Medical Center, Okayama, Japan; ${ }^{2}$ Department of
Respiratory Surgery, National Hospital Organization Okayama Medical Center, Okayama, Japan. Email: h_hisao_430@yahoo.co.jp

Received March 20 ${ }^{\text {th }}, 2013$; revised April 22 ${ }^{\text {nd }}, 2013$; accepted April 30 ${ }^{\text {th }}, 2013$

Copyright (C) 2013 Hisao Higo et al. This is an open access article distributed under the Creative Commons Attribution License, which permits unrestricted use, distribution, and reproduction in any medium, provided the original work is properly cited.

\begin{abstract}
Background: We reported our experience with thymic carcinomas and review their clinical features, treatment strategies, and prognoses. Methods: From April 1998 to November 2012, 11 patients pathologically diagnosed with thymic carcinoma and treated in our hospital were investigated. Results: There were 7 men and 4 women, with a median age of 62 years (range, 35 - 72). According to the Masaoka staging system, 3 patients had stage II, 1 stage III disease, 3 stage IVa disease and 4 stage IVb disease. Ten patients had squamous cell carcinoma, whereas 1 had large cell neuroendocrine carcinoma (LCNEC). We performed surgery or multimodality therapy including surgery as the initial therapy for 8 patients. Of the non-surgical cases, 1 patient received chemoradiotherapy and survived for over 6 years without recurrence, whereas 2 received palliative care. Three of 4 patients who underwent complete resection survived without disease recurrence, whereas only 1 patient with LCNEC survived in the incomplete resection group. Multimodality therapy with cisplatin and docetaxel was provided to 3 patients, and recurrence has not been observed in any of the cases. Conclusions: Favorable outcomes could be achieved in patients with thymic carcinoma who underwent intensive treatment. In particular, surgery combined with cisplatin and docetaxel plus thoracic irradiation may be an attractive approach for thymic carcinoma.
\end{abstract}

Keywords: Thymic Carcinoma; Multimodality Treatment; Cisplatin; Docetaxel

\section{Introduction}

Thymic carcinoma is a rare tumor arising from the thymic epithelium that accounts for approximately $14.1 \%$ of thymic epithelial neoplasms and differs from thymoma with respect to morphological and biological features. The most common histologic type in Japan is squamous cell carcinoma [1]. The prognosis of thymic carcinoma is worse than that of thymoma, with a 5-year survival rate of 33.3\% to $50.5 \%$, which correlates with the Masaoka staging system [1,2]. Unfortunately, $80 \%$ to $90 \%$ of cases are diagnosed at an advanced stage (stage III or IV) [1,3]. Treatment with surgery, radiotherapy, and chemotherapy has been described in some reports, but there is no consensus as to which modality or combined modality is the gold standard. We report our experience with 11 cases of thymic carcinoma and review their clinical features, treatment, and prognoses.

\section{Patients and Methods}

From April 1998 to November 2012, 11 patients were pathologically diagnosed with thymic carcinoma and treated in our hospital. We retrospectively reviewed their clinical features, treatment, and prognoses. The clinical or pathological stage of the disease was determined according to the staging system described by Masaoka et al. [4]. The pathological diagnosis of thymic carcinoma was established according to the histopathological criteria proposed by the World Health Organization (WHO) [5].

Overall survival time was calculated from the date of diagnosis until death or the last follow-up visit using the Kaplan-Meier method. Statistical analyses were performed using SPSS version 20 for Windows (IBM). 


\section{Results}

\subsection{Patient Characteristics}

The study included 7 men and 4 women, with a median age of 62 years (range, 35 - 72). According to the Masaoka staging system, 3 patients had stage II disease, 1 stage III disease, 3 stage IVa disease and 4 stage IVb disease. Pathologically, 10 patients had squamous cell carcinoma, whereas 1 had large cell neuroendocrine carcinoma (LCNEC). We performed surgery or multimodality therapy including surgery as the initial therapy for 8 patients. Of the non-surgical cases, 1 patient received chemoradiotherapy, whereas 2 received palliative care.

\subsection{Treatment Strategies and Clinical Outcomes}

Table 1 summarizes all cases with respect to their initial treatment and respective outcomes. One patient (case 7) from the non-surgery group received pericardial drainage and died 21 months after the diagnosis. A second patient (case 8) was treated with radiotherapy to the site of bone metastasis followed by chemotherapy and eventually died 23 months later. Another patient (case 9) received chemotherapy consisting of cisplatin and docetaxel with concurrent thoracic irradiation in accordance with the OLCSG 0007 protocol [6]; this patient is still alive 6 years later without disease progression (Figure 1).

In the surgical intervention group, 1 patient received surgery alone, 2 patients received surgery and adjuvant radiotherapy, 3 patients received surgery with adjuvant chemotherapy and subsequent radiotherapy, and 2 patients received preoperative chemoradiotherapy, surgery, and adjuvant chemotherapy. Complete resection was per- formed in 4 of the 8 cases (Table 2). In the complete resection group, 3 of 4 patients were alive and disease free at the time of reporting, whereas the other patient (case 2) had recurrence of pleural dissemination. In the incomplete resection group, 1 patient with LCNEC was alive with no evidence of disease following postoperative radiotherapy and chemotherapy consisting of carboplatin and etoposide at the time of reporting.

However, the other 3 patients experienced disease recurrence. As shown in Table 2, better outcomes were achieved in patients at an early Masaoka stage and in those who underwent complete resection together with radiotherapy and chemotherapy.

Table 3 shows the site of disease recurrence in each patient who received surgery. Manifestations of disease recurrence were dissemination to the pleura, supraclavicular lymph nodes, and lungs. Seven of the 11 patients are still alive. The median survival time and 5-year survival rate were 63.7 months and $58.3 \%$, respectively.

\subsection{Treatment Outcomes According to Chemotherapy Regimens}

Recurrence was not observed in any of the 3 patients treated with this regimen (cisplatin and docetaxel) at the time of reporting. As mentioned above, 1 of the patients was alive for over 6 years with no further need for surgery. In another patient, complete resection was achieved by performing preoperative chemoradiotherapy. In contrast, 2 of 3 patients treated with cisplatin alone or with the adriamycin, cisplatin, vincristine, and cyclophosphamide (ADOC) regimen experienced recurrent diseases (Table 4).

Table 1. Summary of 11 cases of thymic carcinoma.

\begin{tabular}{|c|c|c|c|c|c|c|c|c|c|c|}
\hline \multirow{2}{*}{ Case } & \multirow{2}{*}{ Age } & \multirow{2}{*}{ Sex } & \multirow{2}{*}{ Histology } & \multirow{2}{*}{ Stage } & \multicolumn{3}{|c|}{ Initial treatment } & \multirow{2}{*}{ Recurrence } & \multirow{2}{*}{ DFS(M) } & \multirow{2}{*}{ Status } \\
\hline & & & & & Surgery & RT & $\mathrm{Cx}$ & & & \\
\hline 1 & 66 & $\mathrm{~F}$ & LCNEC & II & + & + & + & No & 43.1 & Alive \\
\hline 2 & 35 & M & $\mathrm{Sq}$ & II & + & + & - & Yes & 8.0 & Alive \\
\hline 3 & 60 & M & $\mathrm{Sq}$ & II & + & + & - & No & 20.6 & Alive \\
\hline 4 & 70 & $\mathrm{~F}$ & Sq & III & + & + & + & Yes & 17.9 & Dead \\
\hline 5 & 56 & $\mathrm{~F}$ & $\mathrm{Sq}$ & IVa & + & - & - & Yes & 26.5 & Alive \\
\hline 6 & 67 & M & $\mathrm{Sq}$ & IVa & + & + & + & Yes & 32.9 & Dead \\
\hline 7 & 42 & M & $\mathrm{Sq}$ & IVa & - & - & - & - & - & Dead \\
\hline 8 & 62 & M & Sq & $\mathrm{IVb}$ & - & + & - & - & - & Dead \\
\hline 9 & 70 & M & $\mathrm{Sq}$ & $\mathrm{IVb}$ & - & + & + & No & 80.7 & Alive \\
\hline 10 & 72 & M & $\mathrm{Sq}$ & $\mathrm{IVb}$ & + & + & + & No & 46.2 & Alive \\
\hline 11 & 59 & F & $\mathrm{Sq}$ & $\mathrm{IVb}$ & + & + & + & No & 14.8 & Alive \\
\hline
\end{tabular}

Abbreviations: DFS, disease-free survival; LCNEC, large cell neuroendocrine carcinoma; Sq, squamous cell carcinoma; RT, radiotherapy; Cx, chemotherapy. 


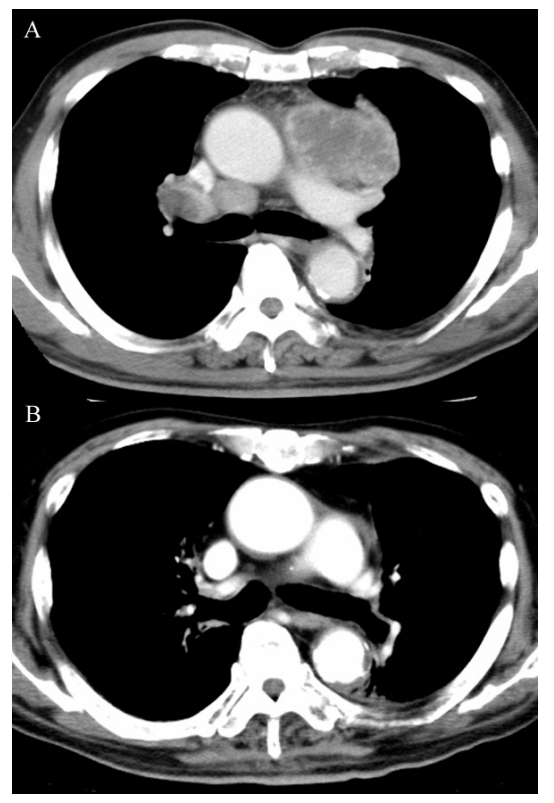

Figure 1. Computed tomography scan of the chest in case 9 showed a bulky mass in the anterior mediastinum with mediastinal and right hailer lymphadenopathy in April 2006 (A); Complete remission was achieved by treatment with cisplatin and docetaxel accompanied by concurrent thoracic irradiation, and the patient was alive for over 6 years without disease progression at the time of reporting (B).

Table 2. Outcomes according to clinical stage and treatment modality.

\begin{tabular}{llccc}
\hline \multirow{2}{*}{ Stage } & & \multicolumn{2}{c}{ Recurrence } & \multirow{2}{*}{ Total } \\
\cline { 3 - 4 } & II & $2(67 \%)$ & $1(33 \%)$ & 3 \\
\multirow{3}{*}{ Resection } & III or IV & $3(38 \%)$ & $5(62 \%)$ & 8 \\
& Complete & $3(75 \%)$ & $1(25 \%)$ & 4 \\
\multirow{3}{*}{ Radiotherapy } & Incomplete & $1(25 \%)$ & $3(75 \%)$ & 4 \\
& Non-Resection & $1(33 \%)$ & $2(67 \%)$ & 3 \\
\multirow{2}{*}{ Chemotherapy } & + & $4(50 \%)$ & $4(50 \%)$ & 8 \\
& - & $0(0 \%)$ & $3(100 \%)$ & 3 \\
& + & $4(67 \%)$ & $2(33 \%)$ & 6 \\
& - & $1(20 \%)$ & $4(80 \%)$ & 5 \\
\hline
\end{tabular}

Table 3. Recurrent sites in patients who underwent surgical resection.

\begin{tabular}{lllc}
\hline & \multicolumn{1}{c}{ Recurrence site } & Status & OS (M) \\
\hline 2 & Pleura & alive & 40.3 \\
4 & Supraclavicular Lymph Node & dead & 64.6 \\
5 & Pleura & alive & 176 \\
6 & Lung & dead & 53.0 \\
\hline
\end{tabular}

Abbreviation: OS, overall survival.

\section{Discussion}

Surgery is considered to be the mainstay of treatment for thymic carcinoma. Complete resection is particularly important [7]. Kondo et al. showed 5-year survival rates of $66.9 \%, 29.8 \%$, and $19.4 \%$ in the complete resection group, incomplete resection group, and non-surgery groups, respectively [1]. In accordance with these findings, 3 of our 4 patients who underwent complete resection survived without recurrence, highlighting the importance of complete resection. However, extensive invasion or metastasis at diagnosis is a frequent finding. Even with complete resection, $50 \%$ of patients are reported to experience disease recurrence [1]. As a result, treatment with multiple modalities including surgery, radiotherapy, and chemotherapy has been attempted [8], but without general consensus on the optimal approach.

Multimodality treatment is expected to be more effective, and some studies have reported its high efficacy [3, 8]. However, other studies have suggested that adjuvant radiotherapy and adjuvant chemotherapy do not contribute to a favorable prognosis [1,9]. In our study, local recurrence was detected in only 1 of the 8 patients who underwent mediastinal radiotherapy. However, 3 of these patients experienced distant metastases. Therefore, we speculate that radiotherapy has potential for good in-field control but is insufficient to control distant metastases. In contrast, chemotherapy is effective not only on primary lesions but also on distant metastases. Platinum-based regimens have been widely used, and the response rate

Table 4. Treatment outcome according to chemotherapy regimen.

\begin{tabular}{|c|c|c|c|c|c|c|c|c|}
\hline \multirow{2}{*}{ Case } & \multirow{2}{*}{ Histology } & \multirow{2}{*}{ Stage } & \multicolumn{3}{|c|}{ Initial Treatment } & \multirow{2}{*}{ Recurrence } & \multirow{2}{*}{ DFS(M) } & \multirow{2}{*}{ Status } \\
\hline & & & Surgery & RT & Regimen & & & \\
\hline 1 & LCNEC & II & + & + & $\mathrm{CE}$ & No & 43.1 & Alive \\
\hline 3 & $\mathrm{Sq}$ & III & + & + & CDDP & Yes & 20.6 & Alive \\
\hline 6 & $\mathrm{Sq}$ & IVa & + & + & ADOC, 5FU & Yes & 32.9 & Dead \\
\hline 9 & $\mathrm{Sq}$ & $\mathrm{IVb}$ & - & + & DP & No & 80.7 & Alive \\
\hline 10 & $\mathrm{Sq}$ & $\mathrm{IVb}$ & + & + & DP & No & 46.2 & Alive \\
\hline 11 & $\mathrm{Sq}$ & IVb & + & + & DP & No & 14.8 & Alive \\
\hline
\end{tabular}

Abbreviations: RT, radiotherapy; DFS, disease-free survival; LCNEC, large cell neuroendocrine carcinoma; Sq, squamous cell carcinoma; CE, carboplatin, etoposide; CDDP, cisplatin; ADOC, doxorubicin, cisplatin, vincristine, cyclophosphamide; DP, cisplatin, docetaxel. 
has been reported to be $22 \%$ to $75 \%$ [10-13]. In addition, recent reports have shown the efficacy of third-generation anti-cancer agents. However, we do know that complete response is very rare and that local control in particular is difficult to achieve by means of chemotherapy alone. Therefore, the use of multimodality therapy is worthwhile when aiming to cure the disease.

Indeed, we treated 3 patients with combination chemotherapy consisting of cisplatin and docetaxel accompanied by radiotherapy, and none of these patients experienced recurrent diseases. Surprisingly, 1 patient (case 9) treated with this regimen and concurrent thoracic irradiation survived for over 6 years without any recurrence, indicating that this chemoradiotherapy controlled the primary lesion and micrometastases and has potential for the cure of thymic carcinomas. Recently, Segawa et al. showed the efficacy of docetaxel and cisplatin with concomitant radiotherapy in locally advanced non-small cell lung cancer (NSCLC) [6]. Therefore, this strategy appears to be an attractive approach for the treatment of thymic carcinoma.

Additionally, because many studies have emphasized the importance of complete resection, preoperative chemoradiotherapy to improve the resectability of the tumor may be a useful strategy. Some studies have evaluated preoperative chemoradiotherapy [14-16], but there is still no definitive consensus regarding this approach. Toyooka et al. recently reported the superiority of induction chemoradiotherapy using cisplatin and docetaxel together with concurrent radiotherapy in comparison with induction chemotherapy for locally advanced NSCLC [17]. In this report, they described that the rate of pathological downstaging was significantly higher in the chemoradiotherapy group than in the chemotherapy group, resulting in significantly longer overall survival and disease-free survival in the chemoradiotherapy group. We also observed good outcomes when we treated patients with preoperative cisplatin and docetaxel in combination with radiotherapy and believe that this preoperative approach may improve tumor resectability.

Our study has some limitations. First, this is simply a study of a series of cases of thymic carcinoma, and the sample size is too small to conclusively determine the efficacy of multimodal approaches. Second, we cannot simply compare each of our cases because of differences in pathological differentiation and clinical stage. Thymic carcinoma is a rare malignant tumor, and therefore, we cannot develop a new strategy based on a study at a single institute. Further studies are warranted to establish evidence for the treatment of thymic carcinoma in a multi-center clinical study setting.

\section{REFERENCES}

[1] K. Kondo and T. Monden, "Therapy for Thymic Epithe- lial Tumors: A Clinical Study of 1320 Patients from Japan," The Annals of Thoracic Surgery, Vol.76, No. 3, 2003, pp. 878-884. doi:10.1016/S0003-4975(03)00555-1

[2] S. Suster and J. Rosai, "Thymic Carcinoma. A Clinicopathologic Study of 60 Patients,” Cancer, Vol. 67, No. 4, 1991, pp. 1025-1032.

doi:10.1002/1097-0142(19910215)67:4<1025::AID-CNC R2820670427>3.0.CO;2-F

[3] K. Ogawa, T. Toita, T. Uno, et al., "Treatment and Prognosis of Thymic Carcinoma: A Retrospective Analysis of 40 Cases,” Cancer , Vol. 94, No. 12, 2002, pp. 3115-3119. doi:10.1002/cncr.10588

[4] A. Masaoka, Y. Monden, K. Nakahara, et al., "Follow-Up Study of Thymomas with Special Reference to Their Clinical Stages,” Cancer, Vol. 48, No. 11, 1981, pp. 2485- 2492.

doi:10.1002/1097-0142(19811201)48:11<2485::AID-CN CR2820481123>3.0.CO;2-R

[5] W. D. Travis, E. Brambilla, H. K. Muller-Hermelink, et al., "World Health Organization Classification of Tumours. Pathology and Genetics of Tumours of the Lung, Pleura, Thymus and Heart," International Agency for Research on Cancer Press, Lyon, 2004, pp. 146 -151.

[6] Y. Segawa, K. Kiura, N. Takigawa, et al., "Phase III Trial Comparing Docetaxel and Cisplatin Combination Chemotherapy with Mitomycin, Vindesine, and Cisplatin Combination Chemotherapy with Concurrent Thoracic Radiotherapy in Locally Advanced Non-Small-Cell Lung Cancer, OLCSG 0007,” Journal of Clinical Oncology, Vol. 28, No. 20, 2010, pp. 3299-3306. doi:10.1200/JCO.2009.24.7577

[7] C. Y. Lee, M. K. Bae, I. K. Park, et al., "Early Masaoka Stage and Complete Resection Is Important for Prognosis of Thymic Carcinoma: A 20-Year Experience at a Single Institution,” European Journal Cardio-Thoracic Surgery, Vol. 36, No. 1, 2009, pp. 159-162. doi:10.1016/j.ejcts.2009.02.019

[8] M. Lucchi, A. Mussi, F. Basolo, et al., "The Multimodality Treatment of Thymic Carcinoma,” European Journal Cardio-Thoracic Surgery, Vol. 19, No. 5, 2001, pp. 566569. doi:10.1016/S1010-7940(01)00666-2

[9] G. Cardillo, F. Carleo, R. Giunti, et al., "Predictors of Survival in Patients with Locally Advanced Thymoma and Thymic Carcinoma (Masaoka Stages III and IVa),” European Journal Cardio-Thoracic Surgery, Vol. 37, No. 4, 2010, pp. 819-823. doi:10.1016/j.ejcts.2009.11.001

[10] G. L. Lemma, J. W. Lee, S. C. Aisner, et al., "Phase II Study of Carboplatin and Paclitaxel in Advanced Thymoma and Thymic Carcinoma," Journal of Clinical Oncology, Vol. 29, No. 15, 2011, pp. 2060-2065. doi:10.1200/JCO.2010.32.9607

[11] T. Koizumi, Y. Takabayashi, S. Yamagishi, et al., "Chemotherapy for Advanced Thymic Carcinoma: Clinical Response to Cisplatin, Doxorubicin, Vincristine, and Cyclophosphamide (ADOC Chemotherapy)," American Journal of Clinical Oncology, Vol. 25, No. 3, 2002, pp. 266268. doi:10.1097/00000421-200206000-00012

[12] S. Igawa, H. Murakami, T. Takahashi, et al., "Efficacy of Chemotherapy with Carboplatin and Paclitaxel for Unre- 
sectable Thymic Carcinoma,” Lung Cancer, Vol. 67, No. 2, 2010, pp. 194-197. doi:10.1016/j.lungcan.2009.03.031

[13] Y. Okuma, Y. Hosomi, Y. Takagi, et al., "Cisplatin and Irinotecan Combination Chemotherapy for Advanced Thymic Carcinoma: Evaluation of Efficacy and Toxicity,” Lung Cancer, Vol. 74, No. 3, 2011, pp. 492-496. doi:10.1016/j.lungcan.2011.05.013

[14] M. Yano, H. Sasaki, T. Yokoyama, et al., “Thymic Carcinoma: 30 Cases at a Single Institution," Journal of Thoracic Oncology, Vol. 3, No. 3, 2008, pp. 265-269. doi:10.1097/JTO.0b013e3181653c71

[15] M. Abiko and T. Satoh. "Effect of Preoperative Concurrent Chemo-Radiotherapy for Thymic Carcinoma,” Kyobu
Geka, Vol. 55, No. 11, 2002, pp. 965-970.

[16] A. Morio, K. Nakahara, Y. Ohse, et al., "Efficacy of Induction Chemoradiotherapy in Thymic Cancer: Report of a Successful Case and Review of the Literature,” International Journal of Clinical Oncology, Vol. 7, No. 3, 2002, pp. 201-204. doi:10.1007/s101470200030

[17] S. Toyooka, K. Kiura, K. Shien, et al., "Induction Chemoradiotherapy Is Superior to Induction Chemotherapy for the Survival of Non-Small-Cell Lung Cancer Patients with Pathological Mediastinal Lymph Node Metastasis," Interactive CardioVascular and Thoracic Surgery, Vol. 15, No. 6, 2012, pp. 954-960. doi:10.1093/icvts/ivs412 